\title{
One-step RT-qPCR assay for ZIKV RNA detection in Aedes aegypti samples: A protocol to study infection \& gene expression during ZIKV infection
}

\section{Ricardo Vieira Araujo}

Boston University Medical Campus

\section{Fabiana Feitosa-Suntheimer}

Boston University Medical Campus

Alexander S. Gold

Boston University Medical Campus

\section{Berlin Londono-Renteria}

Kansas State University

Tonya Michelle Colpitts ( $\nabla$ tmcol@bu.edu )

University of South Carolina School of Medicine

\section{Methodology}

Keywords: Aedes aegypti, Zika virus, one-step qRT-PCR, mosquito gene expression

Posted Date: January 30th, 2020

DOI: https://doi.org/10.21203/rs.2.19173/v2

License: (c) (1) This work is licensed under a Creative Commons Attribution 4.0 International License. Read Full License

Version of Record: A version of this preprint was published at Parasites \& Vectors on March 14th, 2020. See the published version at https://doi.org/10.1186/s13071-020-4002-x. 


\section{Abstract}

Background: Zika virus (ZIKV) is transmitted to humans during the bite of an infected mosquito. In a scenario of globalization and climate change, the frequency of outbreaks has and will increase in areas with competent vectors, revealing a need for continuous improvement of ZIKV detection tools in vector populations. A simple, rapid and sensitive assay for viral detection is qRT-PCR, yet oligos optimized for ZIKV detection in mammalian cells and samples have repeatedly shown high background when used on mosquito RNA. In this work we present a one-step qRT-PCR protocol that allows for the detection of ZIKV in mosquitoes and for the evaluation of gene expression from the same mosquito sample and RNA. This assay is a less expensive qRT-PCR approach than that most frequently used in the literature and has a much lower background, allowing for confident detection.

Methods: Our new oligo design to detect ZIKV RNA included in silico analysis of both viral and mosquito ( Ae. aegypti and Ae. albopictus ) genomes, targeting sequences conserved between Asian and African ZIKV lineages, but not matching Aedes genomes. This assay will allow researchers to avoid nonspecific amplification in insect samples due to viral integration into the mosquito genome, a phenomenon known to happen in wild and colonized populations of mosquitoes. Standard curves constructed with in vitro transcribed ZIKV RNA were used to optimize the sensitivity, efficiency and reproducibility of the assay.

Results: Finally, the assay was used with success to detect both ZIKV RNA in infected mosquitoes and to detect expression of the Defensin A gene, an antimicrobial peptide (AMP) involved in Aedes aegypti immune response to virus infection.

Conclusions: The experimental approach to detect ZIKV RNA in Aedes aegypti presented here has demonstrated to be specific, sensitive and reliable, and additionally it allows for the analysis of mosquito gene expression during ZIKV infection.

\section{Background}

Zika virus (ZIKV) was first isolated in Uganda, from a sentinel rhesus macaque in 1947 [1]. It is an arthropod-borne virus (arbovirus) belonging to the genus Flavivirus of the Flaviviridae family. ZIKV infection in humans usually results in mild disease or asymptomatic infections, however it can develop into to severe symptoms that can be lethal. The symptomatology can include fever, rash, arthritis and/or arthralgia and/or myalgia, conjunctivitis, and fatigue. Neurological complications caused by ZIKV infection were reported in adults (Guillain-Barré syndrome) and neonates (congenital malformations including microcephaly) [2].

Before a major outbreak of Zika cases in 2007 at the Pacific Island of Yap in the Federate States of Micronesia [3], ZIKV infections occurred in Africa and Asia without much attention. In 2015, the first Zika cases were reported in the Americas (Brazil) and quickly spread to more than 20 countries throughout the Caribbean, and South, Central, and North Americas [4, 5, 6, 7]. In 2016-2017, Zika fever autochthonous cases were reported in USA, in the states of Texas and Florida $[8,9]$. 
ZIKV is transmitted to humans by the bite of an infected mosquito. The main vectors associated with transmission in the urban cycle are Aedes aegypti and Aedes albopictus. In the current state of globalization and climate change, the frequency of human disease outbreaks related to arboviruses, including Zika, has increased in urban centers with competent vectors $[10,11,12,13]$, revealing a need for continuous improvement of ZIKV detection in vector populations. In addition, there has been increased research involving ZIKV infection in the mosquito as an improved understanding of both pathogenesis and interactions in the vector will be crucial information. As such, successful detection of ZIKV infection in mosquito cells and samples is an important component of laboratory work involving the virus and vector.

Molecular detection of ZIKV ribonucleic acid (RNA) in mosquitoes can be challenging due to the limited number of primers and probes published $[14,15,16,17,18,19,20,21,22,23,24,25,26,27]$, as well as the presence of viral genetic material integrated in mosquito genomes $[28,29]$ which can reduce the specificity for RNA detections by quantitative reverse transcription polymerase chain reaction (RT-qPCR) [30]. Most of these ZIKV oligos were optimized in mammalian cells and samples and show oftenunresolvable background when used to detect infection in mosquito tissues.

In recent studies utilizing RT-qPCR to detect ZIKV RNA in mosquitoes, which mostly analyzed vector competence, it is possible to observe a methodological trend favoring the utilization of hydrolysis probes as a fluorescent label (83\% of the papers in literature) and the use of viral RNA extraction kits to obtain the RNA templates (56\% of the studies). However the number of studies could actually be higher since some of them do not specify the extraction RNA method used for the experiments $[9,14,16,18,19,21$, $22,23,31,32,33,34,35,36,37,38,39,40,41,42,43,44,45,46,47,48,49,50,51]$. Although this approach has demonstrated to be relatively effective in detecting ZIKV RNA, since the isolation of viral RNA is prioritized, it does not permit the study of the gene expression in mosquito genes during viral infection using the same samples. The study of mosquito gene expression during ZIKV infection could elucidate phenomena not fully understand regarding ZIKV and mosquito interactions, as vector competence varies in mosquito populations infected with ZIKV isolates from different geographical regions $[52,53,54,55,56]$. In addition, these are very expensive approaches, a potential barrier to vector surveillance in developing countries.

In this study, we present a one-step qRT-PCR protocol that both detects ZIKV RNA and can be used to evaluate gene expression from the same sample of infected $A$. aegypti. In order to avoid nonspecific ZIKV RNA detection due to possible viral integration in the mosquito genome, in silico analysis of the $A$. aegypti, A. albopictus and ZIKV genomes were conducted to find sequences conserved between Asian and African ZIKV phylogenetic lineages $[57,58]$ but divergent from Aedes spp genomes. Primers were designed to detect ZIKV RNA using these determined target regions. Primers were tested on in vitro transcribed ZIKV RNA as well as RNA samples from mosquitoes infected with ZIKV, and the positive mosquito samples were used for transcriptional level analysis of Defensin A, an antimicrobial peptide (AMP) involved in Aedes aegypti immune response [59, 60]. 


\section{Material And Methods}

\section{Cell culture and virus growth}

The Vero (ATCC CCL-81) cell line was used for growing ZIKV Puerto Rico-PRVABC59 (a kind gift of Dr. Stephen Higgs, KSU) and MR766 (BEI Resources, NR-50065) strains. Cells were grown at $37^{\circ} \mathrm{C}$ and $5 \% \mathrm{CO}_{2}$ in Dulbecco's Modified Eagle Medium (DMEM) with 10\% heat-inactivated Fetal Bovine Serum (Gemini) and 1\% Penicillin-streptomycin (Gibco). Infected cells were propagated for 5-7 days before supernatant collection and/or RNA extraction.

\section{Mosquitoes rearing and infection}

Aedes aegypti (Rockefeller strain) were used in all experiments. Mosquito colony was maintained in a secure insectary (Arthropod Containment Level 3 - ACL3). The mosquitoes were bred and maintained in a controlled atmosphere $\left(27{ }^{\circ} \mathrm{C}, 80 \%\right.$ relative humidity, and a 12:12 h light:dark cycle). Larvae were fed with powdered fish food (Tetra), and adult mosquitoes had access to $10 \%$ sucrose solution ad libitum. Female mosquitoes (7-14 days old) were infected by blood-feeding using a Hemotek with ZIKV infected cell supernatant mixed with serum-inactivated human blood from healthy donors (ZenBio), in a 1:1 proportion. The final titer for ZIKV PRVABC59 strain, in the blood solution, was $4 \times 10^{6}$ plaque-forming unit (PFU)/mL. Mosquitoes were allowed to blood feed for $30 \mathrm{~min}$, then iceanesthetized, and non-engorged females were removed. At 7 days post infection, the fed mosquito whole bodies were homogenized in lysis buffer (RLT Buffer - Qiagen) supplemented with $\beta$-Mercaptoethanol $(10 \mu \mathrm{L} / \mathrm{mL})$ and stored at $-20{ }^{\circ} \mathrm{C}$ until RNA extraction.

\section{Primers design}

The ZIKV Nonstructural protein 5 (NS5), the polymerase used for viral RNA synthesis [61], was the selected target for primer design. An alignment of the ZIKV NS5 sequence from the 
Puerto Rico strain (GenBank n. MK028857) was performed with ZIKV Brazil-2015 (GenBank n. KU497555), Cambodia/2010 (GenBank n. MK028862), FrenchPolynesia-2014 (GenBank n. MG976700), Senegal-DakAr41524 (GenBank n. KX601166), and MR766 (GenBank n. MK105975) strains, using the Clustal Omega tool (European Bioinformatics Institute - EMBL-EBI - https://www.ebi.ac.uk/Tools/msa/clustalo/). In addition, an alignment of the ZIKV NS5 sequence (PRVABC59 strain - GenBank n. MK028857) was performed with the Aedes aegypti and Aedes albopictus sequences from "EST", "Assembled transcriptome", and "Transcripts" datasets of the VectorBase Bioinformatics Resource (https://www.vectorbase.org/). A highly conserved sequence region between analyzed ZIKV strains, but divergent from $A$. aegypti and $A$. albopictus mosquito genomes, was identified (Figure 1). This sequence was applied as a template to design primers using the Primer-Blast tool (https://www.ncbi.nlm.nih.gov/tools/primer-blast/), the characteristics of the designed primers (NS5-2362F and NS5-2457R) are demonstrated on Table 1.

\section{Table 1 - Primers and probes used in the one-step RT-qPCR assay}




\begin{tabular}{|c|c|c|c|c|c|}
\hline be & $\begin{array}{c}\text { Gene } \\
\text { product }\end{array}$ & Sequence $5^{\prime}-3^{\prime}$ & $\begin{array}{l}\text { Nucleotide } \\
\text { position }\end{array}$ & $\begin{array}{l}\text { Amplicon } \\
\text { size (bp) }\end{array}$ & Reference \\
\hline 7 & NS5 & GACTGGGTTCCAACTGGGAG & $2362-2381$ & 96 & $*$ \\
\hline \multirow[t]{2}{*}{2} & NS5 & ССАСАСТСТGTТCСАСАССА & $2438-2457$ & & \\
\hline & Env & CCGCTGCCCAACACAAG & 1086-1102 & 76 & [15] \\
\hline $\mathrm{C}$ & Env & CCACTAACGTTCTTTTGCAGACAT & $1139-1162$ & & \\
\hline- & Env & AGCCTACCTTGACAAGCAGTCAGACACTCAA & $1107-1137$ & & \\
\hline- & - & TAATACGACTCACTATAGGGAGACCGCTGCCCAACACAAG & - & - & $*$ \\
\hline Г7 & - & TAATACGACTCACTATAGGGAGAGACTGGGTTCCAACTGGGAG & - & - & $*$ \\
\hline & $\begin{array}{l}\text { Defensin } \\
\text { A }\end{array}$ & AACTGCCGGAGGAAACCTAT & $122-141$ & 116 & [62] \\
\hline & $\begin{array}{l}\text { Defensin } \\
\text { A }\end{array}$ & AATGCAATGAGCAGCACAAG & $218-237$ & & \\
\hline & Actin & GAACACCCAGTCCTGCTGACA & $583-603$ & 65 & [31] \\
\hline & Actin & TGCGTCATCTTCTCACGGTTAG & $626-647$ & & \\
\hline & Actin & AGGCCCCGCTCAACCCGAAG & $605-624$ & & \\
\hline
\end{tabular}

* Designed to this study.

1 Primers containing T7 promoter sequence.

2 Primers used in both qRT-PCR methods (TaqMan and Sybr Green).

\section{One-step RT-qPCR assays}

RNA was extracted from infected cells and/or A. aegypti using RNeasy Mini kit (Qiagen) according to manufacturer's instructions, which included the use of the cell lysate biopolymer-shredding system (QIAshredder, Qiagen) and on-column deoxyribonuclease (DNase) digestion using RNase-free DNase I (Qiagen). RNA sample concentrations were measured using NanoDrop Spectrophotometer (ThermoFisher). For ZIKV RNA detection, one-step RT-qPCR assays were performed on a CFX96 Touch Real-Time PCR Detection System (Bio-Rad), using QuantiFast ${ }^{\circledR}$ SYBR ${ }^{\circledR}$ Green RT-PCR or QuantiFast ${ }^{\circledR}$ Probe RT- 
PCR kits (Qiagen) according to manufacturer's instructions. Primers and probes used on RT-qPCR reactions are shown in Table 1. 40 nanograms of total RNA were used as template. The RT-qPCR cycling protocol consisted of an initial complementary deoxyribonucleic acid (cDNA) synthesis step at $50{ }^{\circ} \mathrm{C}$ for $10 \mathrm{~min}$, a denaturation step at 95 ${ }^{\circ} \mathrm{C}$ for $5 \mathrm{~min}$, followed by 50 replication cycles of $95{ }^{\circ} \mathrm{C}$ for $10 \mathrm{~s}$ and $60{ }^{\circ} \mathrm{C}$ for $30 \mathrm{~s}$. When using QuantiFast ${ }^{\circledR}$ SYBR ${ }^{\circledR}$ Green RT-PCR kit, reactions were performed with primers at $400 \mathrm{nM}$ instead of the concentration recommended by manufacturer's ( $1 \mu \mathrm{M})$. The absolute quantification of ZIKV RNA in mosquito samples was obtained using a standard curve constructed from in vitro transcribed RNA (as described below). A. aegypti Actin was used as control to confirm RNA integrity of samples submitted as well as for absolute quantification and normalization of infection results. Amplicon specificity of ZIKV NS5 was evaluated by the melting curve peak $\left(80 \pm 0.5{ }^{\circ} \mathrm{C}\right)$. The specificity of ZIKV NS5 primers was also evaluated by testing them on viral RNA of Dengue virus (DENV-2 NGC strain) obtained from infected Vero cells and A. aegypti mosquitoes, as negative controls. The relative expression level of $A$. aegypti Defensin A was calculated by DeltaDelta CT (cycle threshold) method, using $A$. aegypti Actin as a gene reference. The primers utilized to amplify Defensin A are shown in Table 1.

\section{In vitro transcription of standard RNA}

Five $\mu \mathrm{g}$ of RNA from Vero cells infected with ZIKV MR766 or PRVABC59 strain were used for synthesis of cDNA using SuperScript III Ribonuclease (RNase) H-Reverse Transcriptase (Invitrogen). First strand cDNA was subjected to PCR using DreamTaq deoxyribonucleic acid (DNA) Polymerase (ThermoFisher) with ZIKV 1086-T7/ZIKV 1162c or NS5-2362-T7 F/NS5-2457R primer sets (Table 1). The PCR products, containing T7 promoter sequence on the positive-sense strand, were used as DNA template for in vitro transcription using the Megascript kit (Ambion), according to manufacturer's instructions. The number of single strand RNA copies (molecules/ $\mu$ l) was calculated as described by Faye et al. (2013) [18].

\section{Results}




\section{Identification of conserved ZIKV region divergent from Aedes}

Aiming to design oligos optimized to minimize non-specific amplification in one-step qRTPCR assay that allows the analysis of mosquito gene expression during ZIKV infection in the same samples, the ZIKV NS5 gene sequence of the PRVABC59 strain was submitted for sequence alignment analysis with Ae. aegypti and Ae. albopictus sequences from "EST", "Assembled transcriptome", and "Transcripts" datasets from VectorBase, using the BLAST tool (https://www.vectorbase.org/blast). At that point, PRVABC59 NS5 sequences without similarity to mosquito genomic sequences were aligned with three ZIKV strains from the Asian phylogenetic lineage (Brazil, French Polynesia and Cambodia) and 2 ZIKV strains from African phylogenetic lineage (Uganda-MR766 and Senegal) using ClustalW. At the conclusion of the analysis, a region of 96 nucleotides from ZIKV NS5, highly conserved between ZIKV strains but divergent from Ae. aegypti and Ae. albopictus, was identified and utilized as template to design qRT-PCR primers (Figure 1).

\section{Confirmation of ZIKV detection in mammalian cells}

We performed qRT-PCR analysis using the designed NS5-2362F and NS5-2457R primers with RNA template isolated from Vero cells infected with ZIKV MR766 and PRVABC59 strains. In Figure 2, the amplification plots for reactions using RNA from cells infected with both strains are shown, demonstrating that the NS5 primers can amplify ZIKV strains from Asian and African phylogenetic lineages.

\section{Assay sensitivity, reproducibility and specificity evaluation}

The ZIKV 1086 / ZIKV 1162c primers and the 1107-FAM probe [15] can be considered the gold standard to ZIKV RNA detection in mosquito samples since it has been preferentially used in most recent studies [31, 33, 34, 35, 36, 38, 46, 49, 51]. The ZIKV 1086 and ZIKV 1162c have been successfully used to detect ZIKV RNA in cell lines, using SYBR Green as a 
dye instead the 1107-FAM [63], and could be a less expensive alternative to ZIKV detection in mosquitoes.

However, we observed nonspecific amplification in half of reactions using uninfected mosquito RNA as template, when using those primers in reaction with SYBR Green, while the reactions with NS5 primers did not present nonspecific detection (Table 2).

\section{Table 2 - Primers specificity evaluation in mosquito samples}

\begin{tabular}{ccccc}
\hline Sample & PCR features & 1107-FAM probe & ZIKV 1086 & NS5 primers \\
& & & ZIKV 1162c primers & \\
\hline Uninfected mosquitoes & Positive/Tested & $0 / 4$ & $2 / 4$ & $0 / 4$ \\
& Quantification cycle (Cq) value & - & $30-31$ & - \\
\cline { 2 - 5 } & Melting temperature & - & $78{ }^{\circ} \mathrm{C}$ & - \\
\hline \multirow{2}{*}{ Infected mosquitoes } & Positive/Tested & $4 / 4$ & $4 / 4$ & $4 / 4$ \\
& Quantification cycle (Cq) value & $22-23$ & $20-21$ & $79.5-80{ }^{\circ} \mathrm{C}$ \\
\cline { 2 - 5 } & Melting temperature & - & $49{ }^{\circ} \mathrm{C}$ & $4 / 4$ \\
\hline Positive control & Positive/Tested & $4 / 4$ & 17 & $18-19$ \\
& Quantification cycle (Cq) value & 20 & $79{ }^{\circ} \mathrm{C}$ & $80{ }^{\circ} \mathrm{C}$ \\
\hline
\end{tabular}

Total RNA from control and ZIKV-infected (PRVABC59 strain) whole mosquitoes were extracted 7 days post infection, and used as template for qRT-PCR reactions, using 1107-FAM probe and NS5-2362F/NS5-2457R or ZIKV 1086/ZIKV 1162c primers. Positive controls consisted of Total RNA from Vero cells infected with ZIKV PRVABC59 strain.

Once ZIKV 1086 / ZIKV 1162c primers were designed to detect ZIKV RNA in human samples, the observed nonspecific amplifications could be caused by similarities between mosquito and viral sequences. Then, we performed the alignment of the sequence amplified by ZIKV 1086 / ZIKV 1162c primers and Aedes aegypti sequences, using the BLAST tool (https://blast.ncbi.nlm.nih.gov/Blast.cgi). In Figure 3 are demonstrated the identities found in four of many significant alignments obtained between the primers annealing sites and mRNA sequences of Aedes aegypti. 
The results shown in Table 2 and Figure 3 demonstrate the importance of optimization with primer design to detect ZIKV RNA in mosquito samples.

Positive single strand RNAs, transcribed in vitro, were used to create standard curves to assess the limit of detection, efficiency and reproducibility of the RT-qPCR assay using NS52362F and NS5-2457R primers and SYBR Green as fluorescent dye. The gold standard used in the literature (ZIKV 1086 / ZIKV 1162c primers and 1107-FAM as the dye) was used in comparison; referred to in the paper as "1107-FAM probe". The detection of the RT-qPCR reactions was linear over 6 ten-fold dilutions $\left(10^{9}\right.$ to $10^{4}$ copies/reaction) using synthetic RNA with sequences from the MR766 and PRVABC59 strains (Figure 4).

The coefficient of determination $\left(\mathrm{R}^{2}\right)$ from synthetic RNA standard curves was calculated to assess the efficiency of reactions using NS5-2362F and NS5-2457R primers, compared to the gold standard (1107-FAM probe). The results show that reactions using the NS5 primers are highly efficient $\left(\mathrm{R}^{2}=0.99\right)$ in the detection of RNA sequences from both ZIKV strains tested (MR766 and PRVABC59) (Figure 4). The determination coefficients obtained in reactions using 1107-FAM probe were 0.99 and 0.97 to MR766 and PRVABC59 strains, respectively.

To further assess the efficiency and reproducibility of this assay, intra and inter assay coefficients of variation (CV) were calculated from quantification cycle (Cq) values obtained using $10^{8}$ copies/reaction of in vitro transcribed RNA, from ZIKV MR766 and PRVABC59 strains. These data are shown in Figure 5. The intra assay coefficients of variation for reactions using NS5 primers were 1.3 $\pm 0.1 \%$ (MR766 strain) and 1.5 $\pm 0.5 \%$ (PRVABC59 strain) while the values obtained using 1107-FAM probe were $1.0 \pm 0.2 \%$ (MR766 strain) and 1.9 $\pm 1.8 \%$ (PRVABC59 strain). The inter assay variation for reactions using NS5 primers were 2.2 and 1.7\% for sequences from MR766 and PRVABC59 strains, respectively. When using 1107-FAM probe, the inter assay variations were 1.3\% for MR766 strain and $1.2 \%$ for PRVABC59 strain. 
The data shown in Figures 4 and 5 show that the developed assay is highly reproducible and efficient, and this assay has the same level of sensitivity as the gold standard used in literature (1107-FAM probe).

\section{Assay detects same levels of ZIKV infection than the gold standard in mosquito samples}

The NS5-2362F and NS5-2457R primers were also tested in RT-qPCR reactions using RNA template isolated from infected blood-fed (IBF) female Aedes mosquitoes. Total RNA from ZIKV-infected whole mosquitoes (PRVABC59 strain) was extracted 7 days post-blood feeding. RT-qPCR using the NS5-2362F and NS5-2457R primers showed an infection rate of approximately 47\% (24 of 51 females), with infection levels of $9.9 \times 10^{4} \pm 8.1 \times 10^{4}$ ZIKV RNA copies/ng Total RNA). The mosquito samples were also analyzed using the 1107-FAM (gold standard) and the results demonstrate a highly approximate ZIKV RNA detection level $\left(9.6 \times 10^{4} \pm 8.4 \times 10^{4}\right)$, as well as the same infection rate, with that obtained using NS5 primers (Figure 6).

\section{Defensin A upregulation detected in orally infected Aedes aegypti}

Since the $A$. aegypti antimicrobial peptide Defensin A has a role in the mosquito immune response to dengue, Chikungunya virus and Zika virus infection [64, 65], we chose to measure expression of this gene in our infected mosquitoes as confirmation that our assay allows for both ZIKV infection detection and analysis of gene expression in the same sample. RNA samples which tested positive for ZIKV were used in additional qRT-PCR analysis to evaluate the transcription of the gene Defensin A, data in Figure 7. The results showed that from the ZIKV-positive mosquitoes Defensin A was upregulated in 69.6\% (16 females), downregulated in $8.7 \%$ ( 2 females), and constitutive in $21.7 \%$ (5 females). In samples that displayed upregulation, the relative expression for Defensin A ranged from 2.2 to 50.3 in fold-change, with a mean of $10.3 \pm 11.9$ fold-change, corroborating results of Zhao 
et al. (2018) that demonstrate Defensin A upregulation level of approximately 5 fold-change in pooled samples (10 females each) of ZIKV infected females [64].

\section{Discussion}

With increasing outbreaks of ZIKV in humans globally, and subsequent increase in experimental analysis of viral pathogenesis and interactions in the mosquito vector, an effective and reliable method to detect ZIKV infection in mosquito samples is essential. This study has presented a sensitive, efficient, and reproducible assay for the detection of ZIKV RNA in Aedes aegypti, using one-step qRT-PCR and oligos based on the viral NS5 gene sequence. This assay also uniquely allows for the analysis of transcriptional levels of mosquito genes during ZIKV infection from the same samples. The method of ZIKV RNA detection in mosquitoes presented here is based on RT-qPCR using a standard RNA extraction kit, primers designed to avoid nonspecific amplification of the mosquito genome and SYBR green as a fluorescent label. Our protocol is relevant since the gold standard for the detection of ZIKV by qRT-PCR in the literature is based on viral RNA extraction kits and the use of hydrolysis probes as the fluorescent label, which is relatively effective but expensive, and the published primer and probe sets were optimized in assays using mammalian samples.

In recent studies that used qRT-PCR analysis to detect ZIKV RNA in mosquitoes, most used hydrolysis probes as fluorescent labels. More than half of the studies that used fluorescent probes to detect ZIKV RNA also used viral extraction kits and four did not describe how RNA extraction was performed $[9,14$, $16,18,22,23,31,32,33,34,35,36,37,38,39,40,41,42,44,45,46,48,49]$.

At this time, only five studies [19, 21, 24, 43,47] have detected ZIKV RNA in mosquitoes using SYBR green. However, in three of these studies $[24,43,47]$ commercial kits designed to obtain mostly viral RNA from samples were used, which will not allow for analysis of mosquito gene expression from the same sample. In the one study that used a standard extraction kit along with SYBR green did not present any analysis about primers specificity, sensitivity, efficiency or reproducibility [19].

In summary, from recent studies in which qRT-PCR was used to detect ZIKV RNA in mosquitoes, $83 \%$ of studies used hydrolysis probes as fluorescent labels and $56 \%$ describe use of viral RNA extraction kits (considering studies that do not specify extraction RNA method it can reach 70\%). Although this approach has been demonstrated to be effective, it is also expensive. In addition, since the isolation of viral RNA is prioritized, this method does not permit the evaluation of the expression of mosquito genes during ZIKV infection. Studies that analyze ZIKV infection in mosquitoes, for example vector competence analyses, often require the assessment of different tissues at different time-points from individual mosquitoes. This work generates large numbers of samples, each requiring RNA extraction as well as multiple qRT-PCR reactions for sufficient analysis, and all of which using current methods come at great expense. Due to this financial barrier, the method presented in this study is an especially relevant alternative for the inexpensive, rapid and reliable detection of ZIKV in mosquito samples by qRT-PCR. 
Considering that majority of ZIKV outbreaks occur in developing countries, the availability of highly sensitive, but also affordable, assays to detect ZIKV can greatly enhance local vectoral surveillance and disease control. Taking into account the experimental conditions and reagents utilized in this work, the prices of PCR reactions using NS5 primers were $31 \%$ less expensive than using 1107-FAM probe $(2.35$ US\$/sample and 3.39 US\$/sample, respectively).

\section{Conclusions}

The experimental approach developed and demonstrated in this study can significantly contribute to research efforts in study of mosquito gene expression during ZIKV infection. The assay used to detect ZIKV RNA in Aedes aegypti presented here has been demonstrated to be sensitive, efficient and reproducible for in vitro analysis in the laboratory as well as on infected mosquito samples. In addition, our presented protocol allows for the analysis of mosquito gene expression during ZIKV infection in the same samples and is a much less expensive approach optimized to minimize nonspecific amplification in Aedes spp samples.

\section{Abbreviations}

ZIKV - Zika virus

qRT-PCR - Quantitative reverse transcription polymerase chain reaction

RNA - Ribonucleic acid

AMP - Antimicrobial peptide

DMEM - Dulbecco's Modified Eagle Medium

ACL3 - Arthropod Containment Level 3

PFU - Plaque-forming unit

NS5 - Nonstructural protein 5

EMBL-EBI - European Bioinformatics Institute

DNase - deoxyribonuclease

DENV - Dengue virus

DNA - Deoxyribonucleic acid

cDNA - Complementary deoxyribonucleic acid

CT - Threshold cycle 
RNase - Ribonuclease

CV - Coefficient of variation

Cq - Quantification cycle

IBF - Infected blood-fed

\section{Declarations}

Ethics approval and consent to participate - N/A

Consent for publication - All authors consent of publication of data

Availability of data and material - All relevant data and material is available

Competing interests - The authors declare no competing interests

Funding - NEIDL funding and Boston University Pilot Award

Authors' contributions - RVA and TMC conceived of the project; RVA, FFS, ASG did the experiments and analyzed data; RVA, FFS, ASG, BLR and TMC contributed to manuscript writing, editing and discussion.

Acknowledgements - N/A

\section{References}

1. Dick GW, Kitchen SF, Haddow AJ. Zika virus. I. Isolations and serological specificity. Trans R Soc Trop Med Hyg. 1952;46 5:509-20; doi: 10.1016/0035-9203(52)90042-4.

https://www.ncbi.nlm.nih.gov/pubmed/12995440.

2. Musso D, Gubler DJ. Zika Virus. Clin Microbiol Rev. 2016;29 3:487-524; doi: 10.1128/CMR.00072-15. https://www.ncbi.nlm.nih.gov/pubmed/27029595.

3. Duffy MR, Chen TH, Hancock WT, Powers AM, Kool JL, Lanciotti RS, et al. Zika virus outbreak on Yap Island, Federated States of Micronesia. N Engl J Med. 2009;360 24:2536-43; doi:

10.1056/NEJMoa0805715. https://www.ncbi.nlm.nih.gov/pubmed/19516034.

4. Zika virus outbreaks in the Americas. Wkly Epidemiol Rec. 2015;90 45:609-10. https://www.ncbi.nlm.nih.gov/pubmed/26552108.

5. Campos GS, Bandeira AC, Sardi SI. Zika Virus Outbreak, Bahia, Brazil. Emerg Infect Dis. 2015;21 10:1885-6; doi: 10.3201/eid2110.150847. https://www.ncbi.nlm.nih.gov/pubmed/26401719.

6. Hennessey M, Fischer M, Staples JE. Zika Virus Spreads to New Areas - Region of the Americas, May 2015-January 2016. MMWR Morb Mortal Wkly Rep. 2016;65 3:55-8; doi:

10.15585/mmwr.mm6503e1. https://www.ncbi.nlm.nih.gov/pubmed/26820163. 
7. Zanluca C, Melo VC, Mosimann AL, Santos GI, Santos CN, Luz K. First report of autochthonous transmission of Zika virus in Brazil. Mem Inst Oswaldo Cruz. 2015;110 4:569-72; doi: 10.1590/007402760150192. https://www.ncbi.nlm.nih.gov/pubmed/26061233.

8. Likos A, Griffin I, Bingham AM, Stanek D, Fischer M, White S, et al. Local Mosquito-Borne Transmission of Zika Virus - Miami-Dade and Broward Counties, Florida, June-August 2016. MMWR Morb Mortal Wkly Rep. 2016;65 38:1032-8; doi: 10.15585/mmwr.mm6538e1. https://www.ncbi.nlm.nih.gov/pubmed/27684886.

9. Martin E, Medeiros MCl, Carbajal E, Valdez E, Juarez JG, Garcia-Luna S, et al. Surveillance of Aedes aegypti indoors and outdoors using Autocidal Gravid Ovitraps in South Texas during local transmission of Zika virus, 2016 to 2018. Acta Trop. 2019;192:129-37; doi: 10.1016/j.actatropica.2019.02.006. https://www.ncbi.nlm.nih.gov/pubmed/30763563.

10. Weaver SC, Reisen WK. Present and future arboviral threats. Antiviral Res. 2010;85 2:328-45; doi: 10.1016/j.antiviral.2009.10.008. https://www.ncbi.nlm.nih.gov/pubmed/19857523.

11. Paz S, Semenza JC. El Nino and climate change--contributing factors in the dispersal of Zika virus in the Americas? Lancet. 2016;387 10020:745; doi: 10.1016/S0140-6736(16)00256-7. https://www.ncbi.nlm.nih.gov/pubmed/26850984.

12. Linthicum KJ, Anyamba A, Tucker CJ, Kelley PW, Myers MF, Peters CJ. Climate and satellite indicators to forecast Rift Valley fever epidemics in Kenya. Science. 1999;285 5426:397-400; doi: 10.1126/science.285.5426.397. https://www.ncbi.nlm.nih.gov/pubmed/10411500.

13. Gould EA, Higgs S. Impact of climate change and other factors on emerging arbovirus diseases. Trans R Soc Trop Med Hyg. 2009;103 2:109-21; doi: 10.1016/j.trstmh.2008.07.025. https://www.ncbi.nlm.nih.gov/pubmed/18799177.

14. Li CX, Guo XX, Deng YQ, Xing D, Sun AJ, Liu QM, et al. Vector competence and transovarial transmission of two Aedes aegypti strains to Zika virus. Emerg Microbes Infect. 2017;6 4:e23; doi: 10.1038/emi.2017.8. https://www.ncbi.nlm.nih.gov/pubmed/28442754.

15. Lanciotti RS, Kosoy OL, Laven JJ, Velez JO, Lambert AJ, Johnson AJ, et al. Genetic and serologic properties of Zika virus associated with an epidemic, Yap State, Micronesia, 2007. Emerg Infect Dis. 2008;14 8:1232-9; doi: 10.3201/eid1408.080287. https://www.ncbi.nlm.nih.gov/pubmed/18680646.

16. Gonzalez MA, Pavan MG, Fernandes RS, Busquets N, David MR, Lourenco-Oliveira R, et al. Limited risk of Zika virus transmission by five Aedes albopictus populations from Spain. Parasit Vectors. 2019;12 1:150; doi: 10.1186/s13071-019-3359-1. https://www.ncbi.nlm.nih.gov/pubmed/30922370.

17. Faye $O$, Faye $O$, Dupressoir A, Weidmann M, Ndiaye M, Alpha Sall A. One-step RT-PCR for detection of Zika virus. J Clin Virol. 2008;43 1:96-101; doi: 10.1016/j.jcv.2008.05.005. https://www.ncbi.nlm.nih.gov/pubmed/18674965.

18. Faye O, Faye O, Diallo D, Diallo M, Weidmann M, Sall AA. Quantitative real-time PCR detection of Zika virus and evaluation with field-caught mosquitoes. Virol J. 2013;10:311; doi: 10.1186/1743-422X-10311. https://www.ncbi.nlm.nih.gov/pubmed/24148652. 
19. Duchemin JB, Mee PT, Lynch SE, Vedururu R, Trinidad L, Paradkar P. Zika vector transmission risk in temperate Australia: a vector competence study. Virol J. 2017;14 1:108; doi: 10.1186/s12985-0170772-y. https://www.ncbi.nlm.nih.gov/pubmed/28599659.

20. Balm MN, Lee CK, Lee HK, Chiu L, Koay ES, Tang JW. A diagnostic polymerase chain reaction assay for Zika virus. J Med Virol. 2012;84 9:1501-5; doi: 10.1002/jmv.23241. https://www.ncbi.nlm.nih.gov/pubmed/22825831.

21. Ryckebusch F, Berthet M, Misse D, Choumet V. Infection of a French Population of Aedes albopictus and of Aedes aegypti (Paea Strain) with Zika Virus Reveals Low Transmission Rates to These Vectors' Saliva. Int J Mol Sci. 2017;18 11; doi: 10.3390/ijms18112384. https://www.ncbi.nlm.nih.gov/pubmed/29125545.

22. Hall-Mendelin S, Pyke AT, Moore PR, Mackay IM, McMahon JL, Ritchie SA, et al. Assessment of Local Mosquito Species Incriminates Aedes aegypti as the Potential Vector of Zika Virus in Australia. PLoS Negl Trop Dis. 2016;10 9:e0004959; doi: 10.1371/journal.pntd.0004959. https://www.ncbi.nlm.nih.gov/pubmed/27643685.

23. Hernandez-Triana LM, Barrero E, Delacour-Estrella S, Ruiz-Arrondo I, Lucientes J, Fernandez de Marco MDM, et al. Evidence for infection but not transmission of Zika virus by Aedes albopictus (Diptera: Culicidae) from Spain. Parasit Vectors. 2019;12 1:204; doi: 10.1186/s13071-019-3467-y. https://www.ncbi.nlm.nih.gov/pubmed/31053164.

24. Tien WP, Lim G, Yeo G, Chiang SN, Chong CS, Ng LC, et al. SYBR green-based one step quantitative real-time polymerase chain reaction assay for the detection of Zika virus in field-caught mosquitoes. Parasit Vectors. 2017;10 1:427; doi: 10.1186/s13071-017-2373-4. https://www.ncbi.nlm.nih.gov/pubmed/28927458.

25. Xu MY, Liu SQ, Deng CL, Zhang QY, Zhang B. Detection of Zika virus by SYBR green one-step realtime RT-PCR. J Virol Methods. 2016;236:93-7; doi: 10.1016/j.jviromet.2016.07.014. https://www.ncbi.nlm.nih.gov/pubmed/27444120.

26. Tappe D, Rissland J, Gabriel M, Emmerich P, Gunther S, Held G, et al. First case of laboratoryconfirmed Zika virus infection imported into Europe, November 2013. Euro Surveill. 2014;19 4; doi: 10.2807/1560-7917.es2014.19.4.20685. https://www.ncbi.nlm.nih.gov/pubmed/24507467.

27. Deng C, Liu S, Zhang Q, Xu M, Zhang H, Gu D, et al. Isolation and characterization of Zika virus imported to China using C6/36 mosquito cells. Virol Sin. 2016;31 2:176-9; doi: 10.1007/s12250-0163778-5. https://www.ncbi.nlm.nih.gov/pubmed/27105638.

28. Palatini U, Miesen P, Carballar-Lejarazu R, Ometto L, Rizzo E, Tu Z, et al. Comparative genomics shows that viral integrations are abundant and express piRNAs in the arboviral vectors Aedes aegypti and Aedes albopictus. BMC Genomics. 2017;18 1:512; doi: 10.1186/s12864-017-3903-3. https://www.ncbi.nlm.nih.gov/pubmed/28676109.

29. Olson KE, Bonizzoni M. Nonretroviral integrated RNA viruses in arthropod vectors: an occasional event or something more? Curr Opin Insect Sci. 2017;22:45-53; doi: 10.1016/j.cois.2017.05.010. https://www.ncbi.nlm.nih.gov/pubmed/28805638. 
30. Castro MG, Nogueira RM, Filippis AM, Ferreira AA, Lima Mda R, Faria NR, et al. Dengue virus type 4 in Niteroi, Rio de Janeiro: the role of molecular techniques in laboratory diagnosis and entomological surveillance. Mem Inst Oswaldo Cruz. 2012;107 7:940-5; doi: 10.1590/s0074-02762012000700017. https://www.ncbi.nlm.nih.gov/pubmed/23147153.

31. Wu P, Sun P, Nie K, Zhu Y, Shi M, Xiao C, et al. A Gut Commensal Bacterium Promotes Mosquito Permissiveness to Arboviruses. Cell Host Microbe. 2019;25 1:101-12 e5; doi:

10.1016/j.chom.2018.11.004. https://www.ncbi.nlm.nih.gov/pubmed/30595552.

32. Richard V, Paoaafaite T, Cao-Lormeau VM. Vector Competence of French Polynesian Aedes aegypti and Aedes polynesiensis for Zika Virus. PLoS Negl Trop Dis. 2016;10 9:e0005024; doi: 10.1371/journal.pntd.0005024. https://www.ncbi.nlm.nih.gov/pubmed/27654962.

33. Nunez Al, Talavera S, Aranda C, Birnberg L, Rivas R, Pujol N, et al. European Aedes caspius mosquitoes are experimentally unable to transmit Zika virus. Parasit Vectors. 2019;12 1:363; doi: 10.1186/s13071-019-3620-7. https://www.ncbi.nlm.nih.gov/pubmed/31345269.

34. Main BJ, Nicholson J, Winokur OC, Steiner C, Riemersma KK, Stuart J, et al. Vector competence of Aedes aegypti, Culex tarsalis, and Culex quinquefasciatus from California for Zika virus. PLoS Negl Trop Dis. 2018;12 6:e0006524; doi: 10.1371/journal.pntd.0006524. https://www.ncbi.nlm.nih.gov/pubmed/29927940.

35. Magalhaes T, Robison A, Young MC, Black WCt, Foy BD, Ebel GD, et al. Sequential Infection of Aedes aegypti Mosquitoes with Chikungunya Virus and Zika Virus Enhances Early Zika Virus Transmission. Insects. 2018;9 4; doi: 10.3390/insects9040177. https://www.ncbi.nlm.nih.gov/pubmed/30513725.

36. Liu Y, Liu J, Du S, Shan C, Nie K, Zhang R, et al. Evolutionary enhancement of Zika virus infectivity in Aedes aegypti mosquitoes. Nature. 2017;545 7655:482-6; doi: 10.1038/nature22365. https://www.ncbi.nlm.nih.gov/pubmed/28514450.

37. Jansen S, Heitmann A, Luhken R, Jost H, Helms M, Vapalahti O, et al. Experimental transmission of Zika virus by Aedes japonicus japonicus from southwestern Germany. Emerg Microbes Infect. 2018;7 1:192; doi: 10.1038/s41426-018-0195-x. https://www.ncbi.nlm.nih.gov/pubmed/30482893.

38. Izquierdo-Suzan M, Zarate S, Torres-Flores J, Correa-Morales F, Gonzalez-Acosta C, Sevilla-Reyes EE, et al. Natural Vertical Transmission of Zika Virus in Larval Aedes aegypti Populations, Morelos, Mexico. Emerg Infect Dis. 2019;25 8:1477-84; doi: 10.3201/eid2508.181533. https://www.ncbi.nlm.nih.gov/pubmed/31310224.

39. Hugo LE, Stassen L, La J, Gosden E, Ekwudu O, Winterford C, et al. Vector competence of Australian Aedes aegypti and Aedes albopictus for an epidemic strain of Zika virus. PLoS Negl Trop Dis. 2019;13 4:e0007281; doi: 10.1371/journal.pntd.0007281. https://www.ncbi.nlm.nih.gov/pubmed/30946747.

40. Heitmann A, Jansen S, Luhken R, Leggewie M, Badusche M, Pluskota B, et al. Experimental transmission of Zika virus by mosquitoes from central Europe. Euro Surveill. 2017;22 2; doi: 10.2807/1560-7917.ES.2017.22.2.30437. https://www.ncbi.nlm.nih.gov/pubmed/28106528. 
41. Gutierrez-Lopez R, Bialosuknia SM, Ciota AT, Montalvo T, Martinez-de la Puente J, Gangoso L, et al. Vector Competence of Aedes caspius and Ae. albopictus Mosquitoes for Zika Virus, Spain. Emerg Infect Dis. 2019;25 2:346-8; doi: 10.3201/eid2502.171123. https://www.ncbi.nlm.nih.gov/pubmed/30666939.

42. Guedes DR, Paiva MH, Donato MM, Barbosa PP, Krokovsky L, Rocha S, et al. Zika virus replication in the mosquito Culex quinquefasciatus in Brazil. Emerg Microbes Infect. 2017;6 8:e69; doi: 10.1038/emi.2017.59. https://www.ncbi.nlm.nih.gov/pubmed/28790458.

43. Evans BR, Kotsakiozi P, Costa-da-Silva AL, loshino RS, Garziera L, Pedrosa MC, et al. Transgenic Aedes aegypti Mosquitoes Transfer Genes into a Natural Population. Sci Rep. 2019;9 1:13047; doi: 10.1038/s41598-019-49660-6. https://www.ncbi.nlm.nih.gov/pubmed/31506595.

44. Dutra HL, Rocha MN, Dias FB, Mansur SB, Caragata EP, Moreira LA. Wolbachia Blocks Currently Circulating Zika Virus Isolates in Brazilian Aedes aegypti Mosquitoes. Cell Host Microbe. 2016;19 6:771-4; doi: 10.1016/j.chom.2016.04.021. https://www.ncbi.nlm.nih.gov/pubmed/27156023.

45. Diagne CT, Diallo D, Faye O, Ba Y, Faye O, Gaye A, et al. Potential of selected Senegalese Aedes spp. mosquitoes (Diptera: Culicidae) to transmit Zika virus. BMC Infect Dis. 2015;15:492; doi: 10.1186/s12879-015-1231-2. https://www.ncbi.nIm.nih.gov/pubmed/26527535.

46. Di Luca M, Severini F, Toma L, Boccolini D, Romi R, Remoli ME, et al. Experimental studies of susceptibility of Italian Aedes albopictus to Zika virus. Euro Surveill. 2016;21 18; doi: 10.2807/15607917.ES.2016.21.18.30223. https://www.ncbi.nlm.nih.gov/pubmed/27171034.

47. Costa-da-Silva AL, loshino RS, Araujo HR, Kojin BB, Zanotto PM, Oliveira DB, et al. Laboratory strains of Aedes aegypti are competent to Brazilian Zika virus. PLoS One. 2017;12 2:e0171951; doi: 10.1371/journal.pone.0171951. https://www.ncbi.nlm.nih.gov/pubmed/28187183.

48. Ciota AT, Bialosuknia SM, Zink SD, Brecher M, Ehrbar DJ, Morrissette MN, et al. Effects of Zika Virus Strain and Aedes Mosquito Species on Vector Competence. Emerg Infect Dis. 2017;23 7:1110-7; doi: 10.3201/eid2307.161633. https://www.ncbi.nlm.nih.gov/pubmed/28430564.

49. Chaves BA, Junior ABV, Silveira KRD, Paz ADC, Vaz E, Araujo RGP, et al. Vertical Transmission of Zika Virus (Flaviviridae, Flavivirus) in Amazonian Aedes aegypti (Diptera: Culicidae) Delays Egg Hatching and Larval Development of Progeny. J Med Entomol. 2019;56 6:1739-44; doi: 10.1093/jme/tjz110. https://www.ncbi.nlm.nih.gov/pubmed/31278737.

50. Caragata EP, Rocha MN, Pereira TN, Mansur SB, Dutra HLC, Moreira LA. Pathogen blocking in Wolbachia-infected Aedes aegypti is not affected by Zika and dengue virus co-infection. PLoS Negl Trop Dis. 2019;13 5:e0007443; doi: 10.1371/journal.pntd.0007443. https://www.ncbi.nlm.nih.gov/pubmed/31107912.

51. Boccolini D, Toma L, Di Luca M, Severini F, Romi R, Remoli ME, et al. Experimental investigation of the susceptibility of Italian Culex pipiens mosquitoes to Zika virus infection. Euro Surveill. 2016;21 35; doi: 10.2807/1560-7917.ES.2016.21.35.30328. https://www.ncbi.nlm.nih.gov/pubmed/27605056. 
52. Weger-Lucarelli J, Ruckert C, Chotiwan N, Nguyen C, Garcia Luna SM, Fauver JR, et al. Vector Competence of American Mosquitoes for Three Strains of Zika Virus. PLoS Negl Trop Dis. 2016;10 10:e0005101; doi: 10.1371/journal.pntd.0005101. https://www.ncbi.nlm.nih.gov/pubmed/27783679.

53. Souza-Neto JA, Powell JR, Bonizzoni M. Aedes aegypti vector competence studies: A review. Infect Genet Evol. 2019;67:191-209; doi: 10.1016/j.meegid.2018.11.009. https://www.ncbi.nlm.nih.gov/pubmed/30465912.

54. Roundy CM, Azar SR, Rossi SL, Huang JH, Leal G, Yun R, et al. Variation in Aedes aegypti Mosquito Competence for Zika Virus Transmission. Emerg Infect Dis. 2017;23 4:625-32; doi: 10.3201/eid2304.161484. https://www.ncbi.nlm.nih.gov/pubmed/28287375.

55. Garcia-Luna SM, Weger-Lucarelli J, Ruckert C, Murrieta RA, Young MC, Byas AD, et al. Variation in competence for ZIKV transmission by Aedes aegypti and Aedes albopictus in Mexico. PLoS Negl Trop Dis. 2018;12 7:e0006599; doi: 10.1371/journal.pntd.0006599. https://www.ncbi.nlm.nih.gov/pubmed/29965958.

56. Chouin-Carneiro T, Vega-Rua A, Vazeille M, Yebakima A, Girod R, Goindin D, et al. Differential Susceptibilities of Aedes aegypti and Aedes albopictus from the Americas to Zika Virus. PLoS Negl Trop Dis. 2016;10 3:e0004543; doi: 10.1371/journal.pntd.0004543. https://www.ncbi.nlm.nih.gov/pubmed/26938868.

57. Lanciotti RS, Lambert AJ, Holodniy M, Saavedra S, Signor Ldel C. Phylogeny of Zika Virus in Western Hemisphere, 2015. Emerg Infect Dis. 2016;22 5:933-5; doi: 10.3201/eid2205.160065. https://www.ncbi.nlm.nih.gov/pubmed/27088323.

58. Faye O, Freire CC, lamarino A, Faye O, de Oliveira JV, Diallo M, et al. Molecular evolution of Zika virus during its emergence in the 20(th) century. PLoS Negl Trop Dis. 2014;8 1:e2636; doi: 10.1371/journal.pntd.0002636. https://www.ncbi.nlm.nih.gov/pubmed/24421913.

59. Magalhaes T, Oliveira IF, Melo-Santos MA, Oliveira CM, Lima CA, Ayres CF. Expression of defensin, cecropin, and transferrin in Aedes aegypti (Diptera: Culicidae) infected with Wuchereria bancrofti (Spirurida: Onchocercidae), and the abnormal development of nematodes in the mosquito. Exp Parasitol. 2008;120 4:364-71; doi: 10.1016/j.exppara.2008.09.003. https://www.ncbi.nlm.nih.gov/pubmed/18809401.

60. Lowenberger C. Innate immune response of Aedes aegypti. Insect Biochem Mol Biol. 2001;31 3:21929; doi: 10.1016/s0965-1748(00)00141-7. https://www.ncbi.nlm.nih.gov/pubmed/11167091.

61. Zhao B, Yi G, Du F, Chuang YC, Vaughan RC, Sankaran B, et al. Structure and function of the Zika virus full-length NS5 protein. Nat Commun. 2017;8:14762; doi: 10.1038/ncomms14762. https://www.ncbi.nlm.nih.gov/pubmed/28345656.

62. Zou Z, Souza-Neto J, Xi Z, Kokoza V, Shin SW, Dimopoulos G, et al. Transcriptome analysis of Aedes aegypti transgenic mosquitoes with altered immunity. PLoS Pathog. 2011;7 11:e1002394; doi: 10.1371/journal.ppat.1002394. https://www.ncbi.nlm.nih.gov/pubmed/22114564. 
63. Londono-Renteria B, Troupin A, Cardenas JC, Hall A, Perez OG, Cardenas L, et al. A relevant in vitro human model for the study of Zika virus antibody-dependent enhancement. J Gen Virol. 2017;98 7:1702-12; doi: 10.1099/jgv.0.000833. https://www.ncbi.nlm.nih.gov/pubmed/28691657.

64. Zhao L, Alto BW, Smartt CT, Shin D. Transcription Profiling for Defensins of Aedes aegypti (Diptera: Culicidae) During Development and in Response to Infection With Chikungunya and Zika Viruses. J Med Entomol. 2018;55 1:78-89; doi: 10.1093/jme/tjx174. https://www.ncbi.nlm.nih.gov/pubmed/28968775.

65. Londono-Renteria B, Grippin C, Cardenas JC, Troupin A, Colpitts TM. Human C5a Protein Participates in the Mosquito Immune Response Against Dengue Virus. J Med Entomol. 2016;53 3:505-12; doi: 10.1093/jme/tjw003. https://www.ncbi.nIm.nih.gov/pubmed/26843451.

\section{Figures}

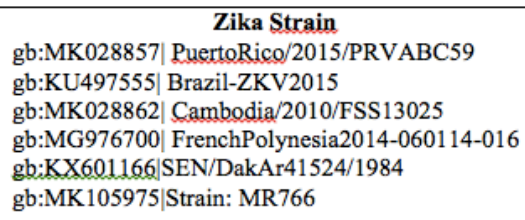

Sequence 5' - 3'

GACTGGGTTCCAACTGGGAGAACTACCTGGTCAATCCATGGAAAGGGAGAATGGATGACCACTGAAGACATGCTTGTGGTGTGGAACAGAGTGTGG GACTGGGTTCCAACTGGGAGAACTACCTGGTCAATCCATGGAAAGGGAGAATGGATGACCACTGAAGACATGCTTGTGGTGTGGAACAGAGTGTGG GACT GGGTTCCAACTGGGAGAACTACCTGGTCAATCCATGGAAAGGGAGAATGGATGACCACT GAAGACATGCTTGTGGTGTGGAACAGAGTGTGG GACTGGGTTCCAACTGGGAGAACTACCTGGTCAATCCATGGAAAGGGAGAATGGATGACCACTGAAGACATGCTTGTGGTGTGGAACAGAGTGTGG GACTGGGTACCAACTGGGAGAACCACCTGGTCAATCCATGGAAAGGGAGAATGGATGACTACTGAGGACATGCTCATGGTGTGGAATAGAGTGTGG GACTGGGTACCAACTGGGAGAACCACCTGGTCAATCCATGGAAAGGGAGAATGGATGACCACTGAGGACATGCTCATGGTGTGGAATAGAGTGTGG

\section{Figure 1}

Alignment of the target amplicon of ZIKV NS5 gene. The nucleotides in red differ from the consensus and the asterisks indicate identity with the consensus sequence on the top. Nucleotides highlighted in yellow correspond to primer (NS5-2362F and NS5-2457R) annealing sites. Clustal Omega tool (EMBL-EBI https://www.ebi.ac.uk/Tools/msa/clustalo/) 


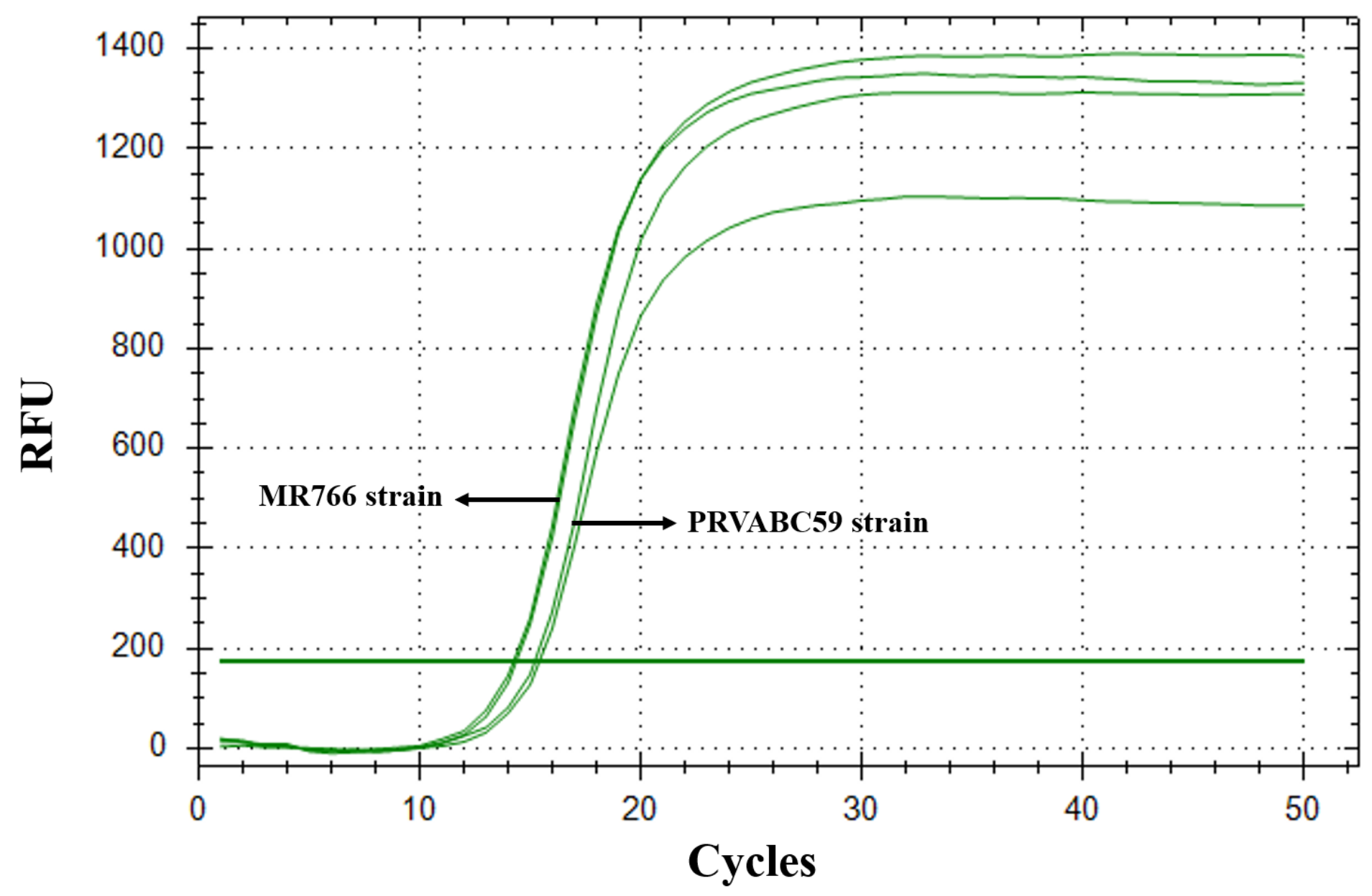

Figure 2

ZIKV strains detected from infected Vero cells. Total RNA from Vero cells infected with ZIKV MR766 and Puerto Rico strains was used as template for one-step RT-qPCR reaction, using NS5-2362F and NS52457R primers. Each sample was tested in duplicate.

\begin{tabular}{|c|c|}
\hline Gene ID & Sequence 5' - 3' \\
\hline gb:MK713748|Organism:Zika yirus|Strain Name:PRVABC59 |Protein Name: polyprotein & CCGCTGCCCAACACAAGGTGAAGCCTACCTTGACAAGCAATCAGACACTCAATATGTCTGCAAAAGAACGTTAGTGG \\
\hline XM_021848756.1 PREDICTED: Aedes aegypti polyhomeotic-p & CCGCTGCCCAACAGCAA- \\
\hline XM_021850239.1 PREDICTED: Aedes aegypti E3 ubiquitin-pr & CCGCTGCCCAACAGCAG-------- \\
\hline XM_021854975.1 PREDICTED: Aedes aegypti centrosomal protein of $135 \mathrm{kDa}$ & --ATGTCTGCAAAAGAAGTCGACAAC \\
\hline XM_021853855.1 PREDICTED: Aedes aegypti maintenance of chromosomes protein 6 & -CGGTCTGCAAAAGAAATCGGCACG \\
\hline
\end{tabular}

\section{Figure 3}

Alignment of the target amplicon amplified by ZIKV 1086 and ZIK 1162c primers with Aedes aegypti mRNA sequences. The nucleotides in bold on Aedes aegypti sequences indicate identities with consensus sequence on the top (Zika virus - PRVABC59 strain: polyprotein). Nucleotides highlighted in yellow correspond to primer (ZIKV 1086 and ZIK 1162c) annealing sites. Sequence highlighted in green correspond to 1107-FAM probe binding site. 
NS5 primers

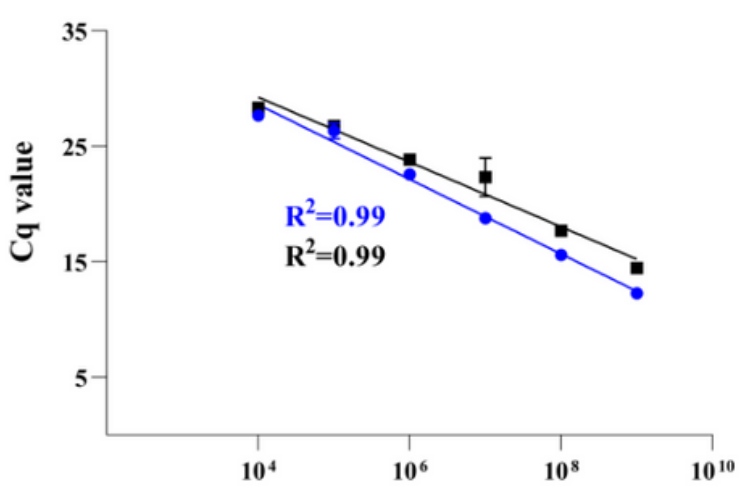

1107-FAM

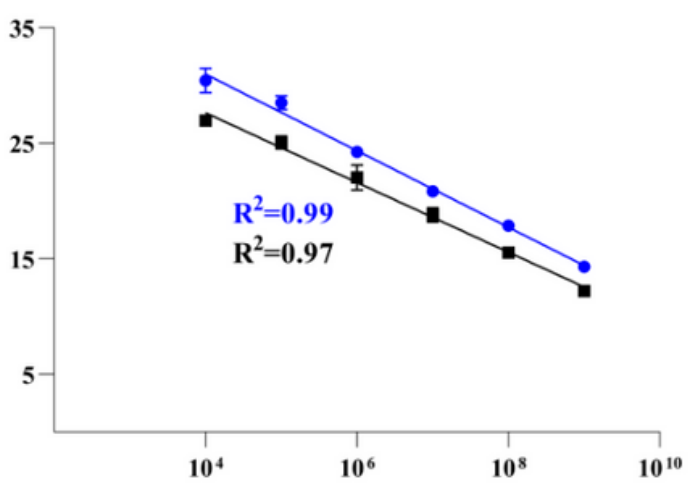

$\rightarrow$ MR766 strain

- PRVABC59 strain

ZIKV synthetic RNA copies

\section{Figure 4}

Limit of Detection and Efficiency of the one-step RT-qPCR assay for ZIKV RNA detection. RNAs transcribed in vitro, containing sequences of NS5 and Env genes from MR766 and PRVABC59 strains, were used as templates for RT-qPCR reactions using hydrolysis probe (1107-FAM) and SYBR Green (NS5$2362 \mathrm{~F}$ and NS5-2457R primers) as fluorescent dyes. Cq values (mean \pm standard deviation) were obtained from two technical replicates, performed in triplicate each. Coefficient of determination (R2) was calculated using GraphPad Prism 8.3.0 software.
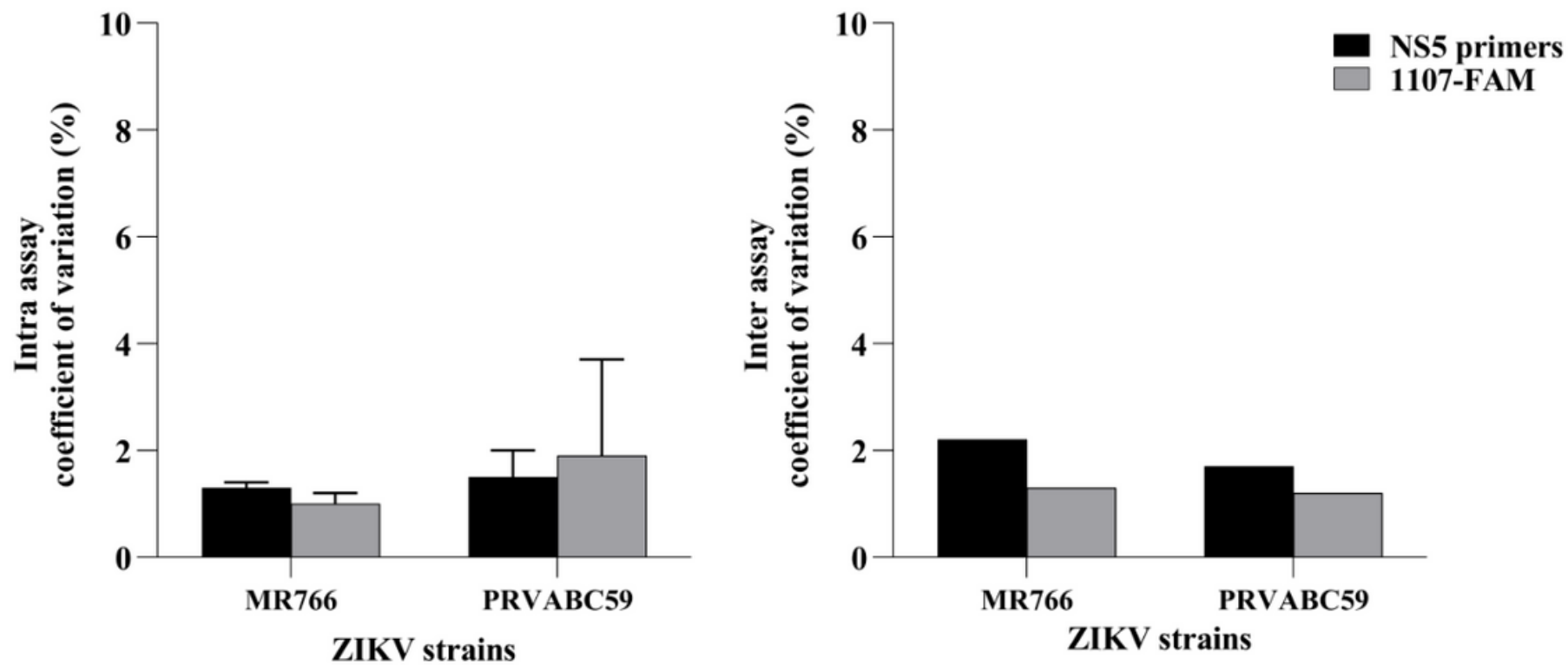

Figure 5

Reproducibility intra and inter assay of the one-step RT-qPCR assay for ZIKV RNA detection. RNAs transcribed in vitro (108 copies/reaction), containing sequences of NS5 and Env genes from MR766 and PRVABC59 strains, were used as templates for RT-qPCR reactions using hydrolysis probe (1107-FAM) and SYBR Green (NS5-2362F and NS5-2457R primers) as fluorescent dyes. Coefficients of variance were 
calculated from three technical replicates, performed in triplicate each. Coefficient of variance $=$ standard deviation/mean $* 100$

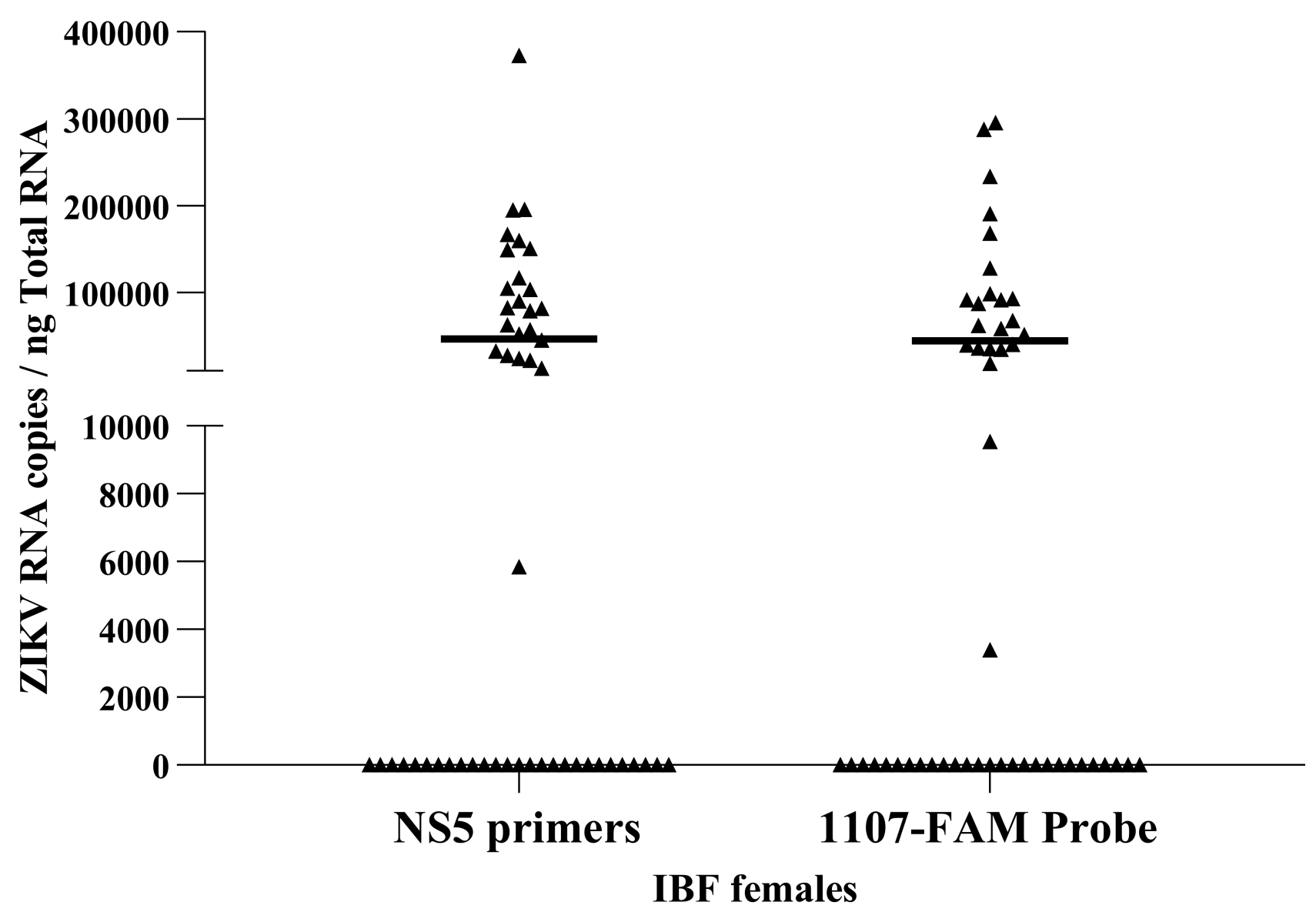

Figure 6

Detection of ZIKV from infected blood-fed (IBF) mosquitoes. Total RNA from ZIKV-infected (PRVABC59 strain) whole mosquitoes were extracted 7 days post infection, and used as template for RT-qPCR reactions, using NS5-2362F/NS5-2457R primers and 1107-FAM probe. The absolute quantification of ZIKV RNA in mosquito samples was obtained using a standard curve constructed from in vitro transcribed RNA. 


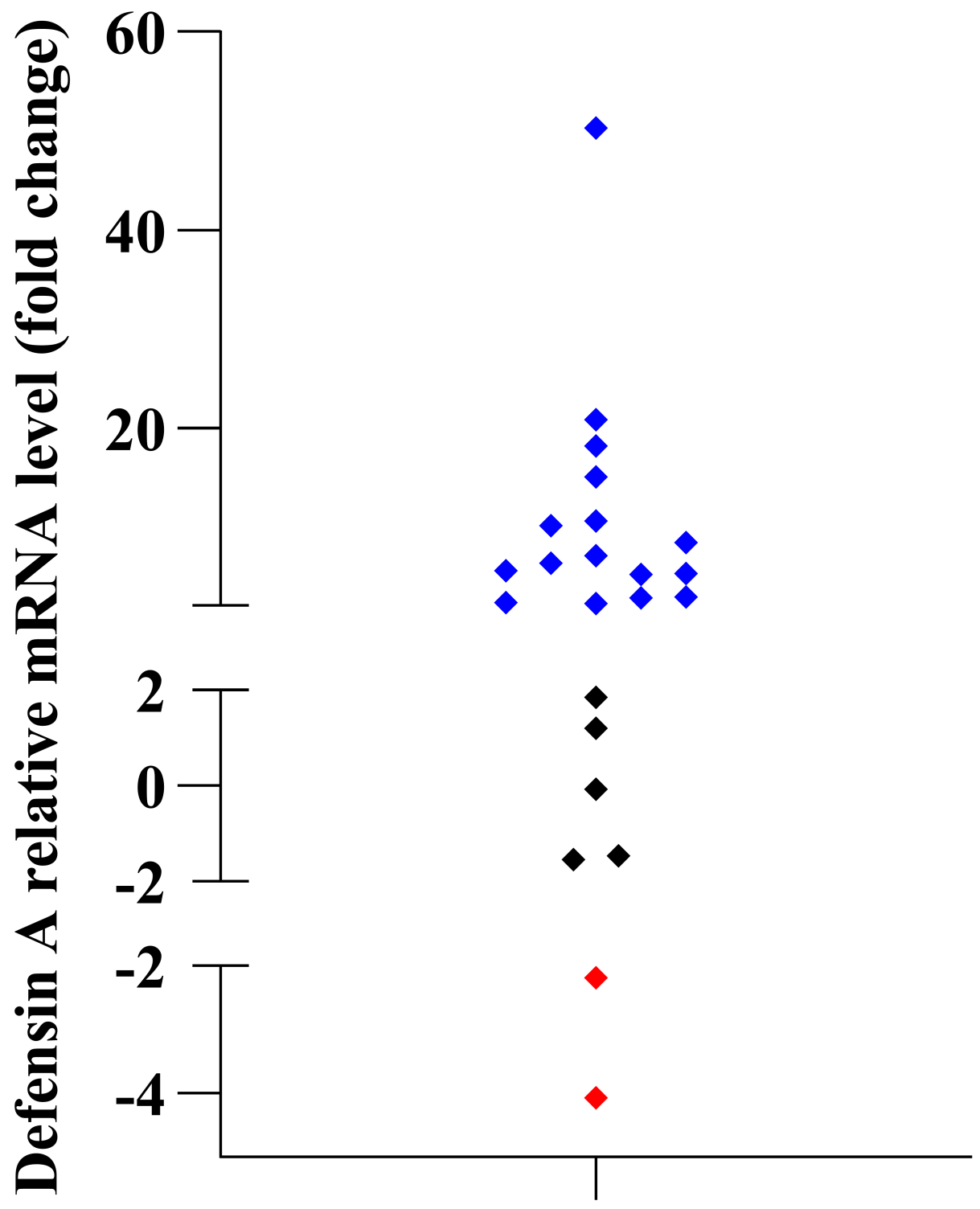

- Upregulated

- Constitutive

- Downregulated

IBF females

Figure 7

Defensin A gene expression in infected blood-fed (IBF) mosquitoes. Samples positive for ZIKV were used for RT-qPCR to evaluate Defensin A transcription levels. Actin was utilized as reference gene. Each sample was tested in duplicate. 